\title{
AW-ISOBIOCLIMAS: APLICACIÓN WEB PARA CONSULTAR LOS ISOBIOCLIMAS DE LA ESPAÑA PENINSULAR Y BALEAR
}

\author{
Ricardo MARCO GARCÍA ${ }^{1}$, Miguel Antonio SARIÑENA USACH², \\ María Soledad LÓPEZ FERNÁNDEZ ${ }^{3}$, María Luisa LÓPEZ FERNÁNDEZ1 \\ ${ }^{1}$ Universidad de Navarra, Depart. de Biología Ambiental, Facultad de Ciencias. \\ ${ }^{2}$ Técnico informático. \\ ${ }^{3}$ Instituto de Estudios Manchegos, CSIC, Ciudad Real. \\ rmarco@unav.es, richardmarco@ono.com, miguelsarinena@miguelsarinena.name, \\ solpfernandez@gmail.com,.mllopez@unav.es
}

\section{RESUMEN}

Para este trabajo se ha preparado una página web, y también un archivo en formato KML, compartido en la misma página web, con la información de los Isobioclimas de la España Peninsular y Balear, publicada en 2015. Para su realización se ha utilizado HTML5, estándar de la W3C para la geolocalización, y Open Layers para generación de mapas web de código abierto. Este formato permite ser leído por múltiples aplicaciones en diferentes sistemas operativos y tanto en dispositivos fijos (ordenadores) como portátiles (móviles y tablets). Así el usuario puede consultar, "in situ", la información del Isobioclima sobre el que se encuentra en el campo. Haciendo un clic de ratón sobre la mancha de Isobioclima, se obtiene más información sobre el mismo.

Los Isobioclimas están definidos por todos los factores bioclimáticos jerarquizados de un área y se expresan con una frase que incluye Macrobioclima, Bioclima, Variante Bioclimática (si la hubiere) y Piso Bioclimático -Termotipo y Ombrotipo- y permiten reconocer las áreas de igual potencialidad biológica. Los geodatos preparados contienen la información de los 72 Isobioclimas encontrados en el territorio de la España Peninsular y Balear.

Palabras clave: Bioclimatología, dispositivos móviles, HTML5, KML, geodatos.

\begin{abstract}
For this work a web page has been prepared, as well as a file in KML format, shared on the same web page, both with information on Isobioclimates of Peninsular Spain and the Balearic Islands, published in 2015. HTML5, W3C standard for geolocation, and Open Layers for generation of open source web mapping, have been used. This format can be read by multiple applications on different operating systems and in both fixed and devices (computers) and portable (mobile and tablets). So the user can consult, "in situ", information about the Isobioclimate of its present position in the field.
\end{abstract}


The Isobioclimates are defined by all hierarchized bioclimatic factors of an área, and are named with a phrase which includes Macrobioclimate, Bioclimate, Biioclimatic Variant (if any) and Bioclimatic

Belt -Thermotype and Ombrotype-. The Isobioclimates allow to recognize he areas with equal biological potentiality. The prepared geodata contain the information on the 72 Isobioclimates found in the territory of Peninsular Spain and the Balearic Islands.

Key words: Bioclimatology, mobile devices, HTML5, KML, geodata

\section{INTRODUCCIÓN}

Los Isobioclimas son la representación climática de la realidad viva, es decir, indican intervalos de parámetros como la temperatura, el confort hídrico, y sus oscilaciones diarias y estacionales, con significación para los seres vivos. Una de las clasificaciones bioclimáticas más reconocidas es la denominada "Worldwide Bioclimatic Classification System”, WBCS (Rivas-Martínez, 2008; Rivas-Martínez et al., 1999, 2011). Con esa metodología se han realizado diversos mapas representando la realidad bioclimática. Cronológicamente, Rivas-Martínez et al., 2002 a y b, publican mapas de Bioclimas y Variantes, y de Termotipos, de Portugal y España; López Fernández et al., en 2003, publican el Mapa Bioclimático de Navarra; y en 2008, los "Macrobioclimas, Bioclimas y Variantes Bioclimáticas de la España Peninsular y Balear"; Piñas et al., en 2008 a y b, publicaron los Termotipos y los Ombrotipos de la España Peninsular y Balear y su cartografía, y Piñas et al., en 2009, "Andalucía: Cartografìa y áreas de sus Bioclimas, Continentalidad, Termotipos y Ombrotipos"; López et al., en 2009, los Isobioclimas de la provincia de Málaga y su Cartografía; y López et al., en 2015, "Mapa Isobioclimático de la España Peninsular y Balear". Mapas bioclimáticos se han realizado también en Portugal (Mesquita y Sousa, 2009), en Cerdeña (Canu et al., 2015) y en toda Italia (Pesaresi et al. 2014).

En los últimos años, la tecnología informática ha llegado a todos los ámbitos de nuestras vidas y, entre ésta, los dispositivos móviles inteligentes, o smartphones, han supuesto una auténtica revolución por sus características. El uso de los dispositivos móviles, con su acceso a internet y sus sensores de localización, amplían las posibilidades para conocer más información sobre nuestra ubicación. La capacidad de comunicación, el acceso a bases de datos "on line" y la capacidad de calcular y transmitir la posición del usuario, hacen de estos dispositivos unas herramientas únicas para transmitir datos referidos a una posición espacial, geodatos. Estas capacidades le han servido al usuario para obtener más información sobre su ubicación. Además de por el geoposicionamiento, el transmitir datos, imágenes o textos de manera remota ha convertido estos dispositivos en eficientes herramientas de toma de datos de campo para investigadores o cualquier usuario del dispositivo. Así, cualquier usuario puede transmitir su posición asociada a un evento con significado biológico o científico, por ejemplo, la presencia de una especie invasora (Plantas Invasoras) o una plaga como es el caso de la aplicación (Mosquito Alert) para detectar la extensión de la plaga del mosquito tigre (Aedes albopictus). Así mismo se han utilizado aplicaciones para móvil para comprobar si hay relación entre los casos de migraña y los cambios de temperatura ambiental (Influence of temperature changes on migraine occurrence in 
Germany, Jörg Scheidt et al., 2013). Para estos usos se han empleado tanto aplicaciones para móvil, como páginas web, por ejemplo, en el caso del proyecto de Cyber Ciudadanos (Cyber Citizens project) que comenzó en 2011 desde la National Science Foundation dedicada a producir herramientas para móvil y web que ayuden a los usuarios y a los científicos, en la captura de información medioambiental con sus dispositivos informáticos. ZamiaDroid es una aplicación que facilita a los usuarios la captura de datos georreferenciados a través de formularios. Se pueden introducir datos textuales (simples o indexados a tesauros), polígonos y/o fotografías, para la definición de proyectos. A su vez, se pueden editar, representar sobre mapas, importar y exportar los datos a hojas de cálculo o bases de datos (ver https://play.google.com-/ store/apps/details?id=uni.projecte). Así mismo, Weather and Climate es una aplicación para motivar a los estudiantes a conocer y explorar el clima y el tiempo atmosférico, permitiendo el cálculo de diversos parámetros climáticos (ver https://play.google. com/store/apps/details?id=com.werkzpublishing.sw.school.weather_climate).

En la actualidad la tecnología digital amplía las posibilidades del papel, y es posible disponer y usar la información de los mapas bioclimáticos en formato digital desde cualquier ordenador, tablet o dispositivo móvil, incluso en pleno trabajo de campo, aprovechando las capacidades de ubicación de esos dispositivos. Coordinar la información bioclimática en formato digital con las capacidades de ubicación de los dispositivos móviles, para poder conocer sobre el terreno el Isobioclima en que nos encontramos, es el objetivo de este trabajo.

\section{MATERIAL Y MÉTODOS}

En este trabajo se ha dispuesto del archivo shapefile de los Isobioclimas de la España Peninsular y Balear, preparado por López et al., 2015. El archivo se ha representado con colores y transparencia para que se pueda visualizar mejor. Además, se le ha añadido un campo con información para que salga la cartela, o pop up, al pinchar en cualquier punto del mapa.

El archivo digital se ha exportado, mediante las herramientas de exportación de ArcGis Desktop, a formato KML, (Keyhole Markup Language) compatible con su visualización en páginas web. Se han creado varias versiones, a mayor y menor resolución, para que se pueda visualizar en la web en dispositivos de distinta capacidad de procesamiento.

La página web se ha realizado con HTML5, el lenguaje utilizado para crear páginas web, y con el último estándar del Consorcio World Wide Web (W3C), que son quienes desarrollan los estándares para su uso en internet, y que permiten la geolocalización. Para el uso de mapas web se han utilizado librerías javascript openlayers. Estos formatos y lenguajes han servido para desarrollar una aplicación que se relaciona con los sensores de nuestro dispositivo móvil, smartphone o PDA (GNSS o sistema de posicionamiento global por satélite) para obtener nuestra posición en el mapa web.

Se ha utilizado un diseño responsivo, para que el tamaño de la página web se adapte al tamaño de pantalla del dispositivo utilizado. Esto permite el acceso a la web desde cualquier dispositivo, móviles o de escritorio. 
Como fondo de mapa se han utilizado dos capas de información gráfica. Para obtener la topografía con las ciudades, carreteras y ríos se ha empleado el mapa libre Open Street Map (OSM) que es un proyecto colaborativo para crear mapas libres y editables. Como fondo de fotografía aérea (ortofotos) se ha utilizado el mapa de PNOA. Es un Servicio de visualización Inspire Teselado: (WMTS) de ortofotos del Instituto Geográfico Nacional (IGN). Mediante el uso de ambos servicios se podrán visualizar los bioclimas sobre fondo topográfico, o sobre ortofotomapa. El uso de estos estándares evita problemas de compatibilidad y hace que la página web se pueda utilizar desde muchos tipos de dispositivos, aunque, por la cantidad de datos gráficos y de información, algunos programas o dispositivos pueden no ser compatibles.

\section{RESULTADOS}

Como resultados tenemos la aplicación web denominada: "AW-Isobioclimas", disponible en: http://www.miguelsarinena.name/isobioclima/

Son mapas web y kml, que permiten la visualización y consulta de los Isobioclimas de la Península Ibérica y Baleares. Pinchando en esa dirección, aparece la portada de la aplicación -ver figura 1-. En esa portada hay tres botones u opciones de consulta a distintas resoluciones, según las capacidades del dispositivo desde el que nos conectemos y de si disponemos, o no, de conexión a internet:

- 1- Mapa web de Isobioclimas a resolución alta, para su consulta "on line" desde Windows o Android.

- 2- Mapa web de Isobioclimas a menor resolución, optimizado para otros dispositivos

- 3- KML para descarga con la misma información, para su uso con o sin conexión.

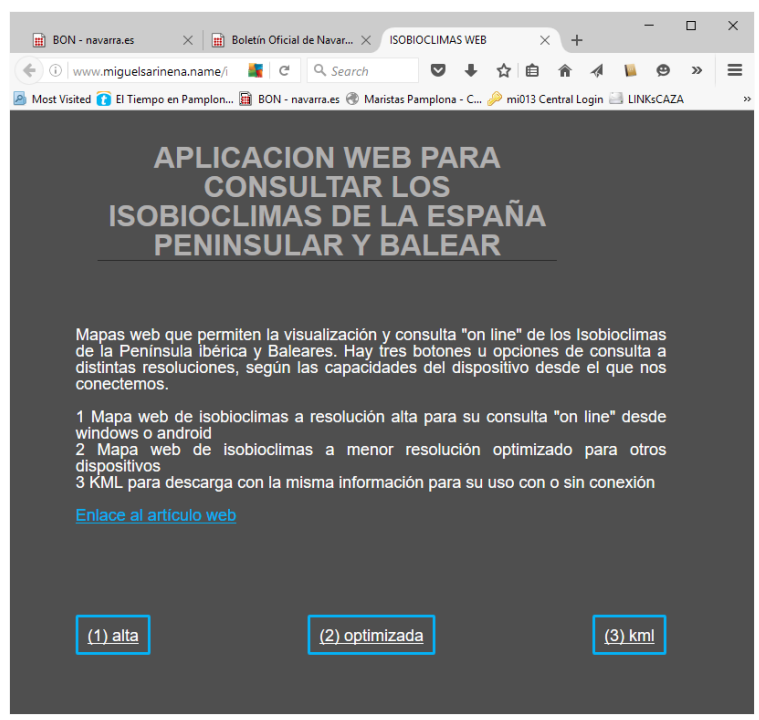

Figura 1: Portada de la aplicación web 
Se ha incluido también un enlace al artículo web donde aparece el mapa y la información de Isobioclimas utilizada en esta aplicación.

La página web completa permite la visualización de los Isobioclimas -figura 2-

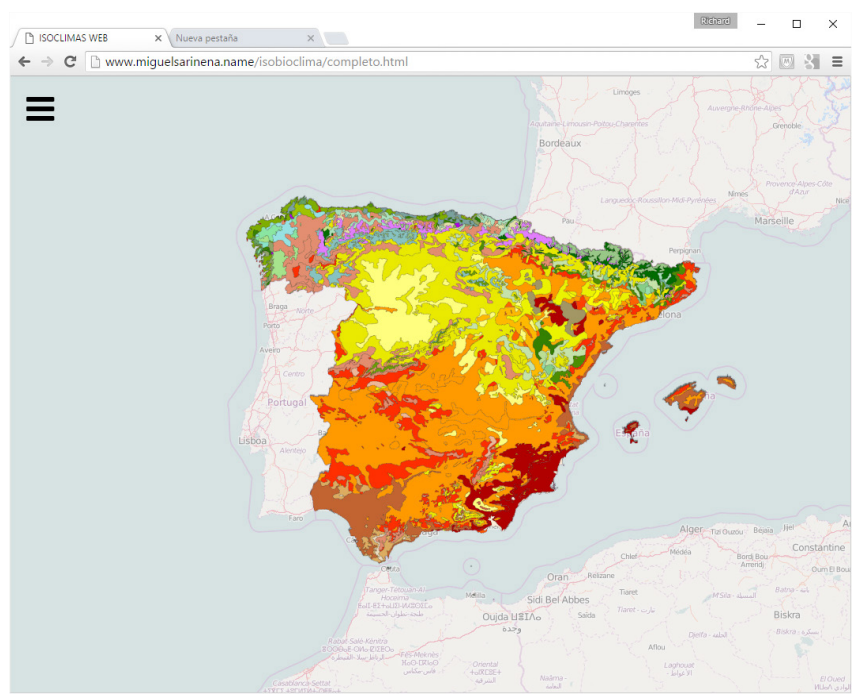

Figura 2: vista de la aplicación web con los Isobioclimas

desde dispositivos fijos (PC con Windows, o MAC) o portátiles con Windows o Android. Los Isobioclimas se visualizan sobre un mapa con fondo topográfico o de fotografía aérea ampliada. Se pueden consultar los datos de referencia de todas las zonas isobioclimáticas haciendo un clic de ratón sobre cada una de ellas. Al cargar la página, el navegador solicitará permiso al usuario para que éste comparta su posición -Figura 3-. Si es así se podrá comprobar el Isobioclima en la ubicación del

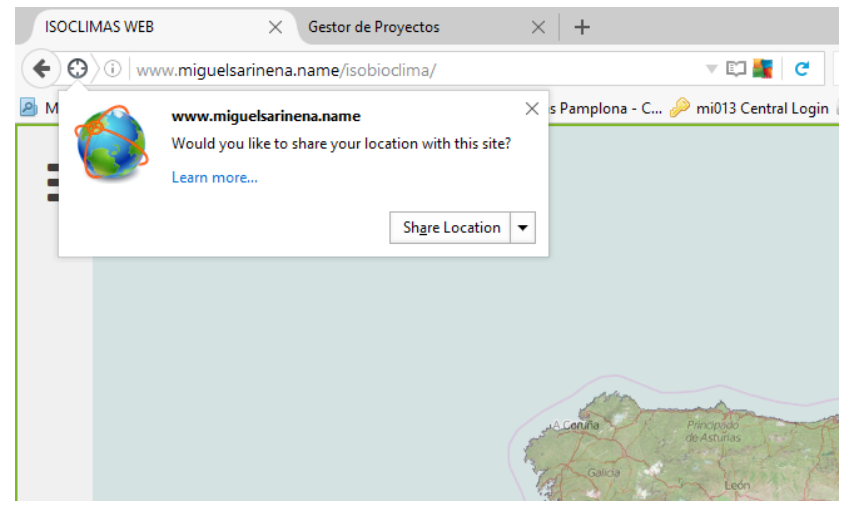

Figura 3: Funcionamiento de la página web de Isobioclimas: solicitud de acceso a la ubicación del usuario desde la página web de la aplicación para obtener su localización en el mapa. 
usuario, opción que es muy interesante si se utiliza dispositivos móviles con sistema de geolocalización, caso en el que podemos comprobar el Isobioclima "in situ", en nuestra posición.

Además del nombre del Isobioclima, al pinchar sobre "Más información", se accede a una web con más datos con el Isobioclima, Macrobioclima, Bioclima, Variante, Termotipo y Ombrotipo.

Aunque, según la documentación de las herramientas web utilizadas, la página web obtenida debía ser de uso universal, se comprobó que algunos dispositivos tenían problemas para cargarla u obtener todas las opciones de la misma: por ello, se ofrece una página web reducida, que utiliza menos recursos, aunque a costa de reducir un poco la precisión en la definición de las manchas de Isobioclimas -figura 4-.
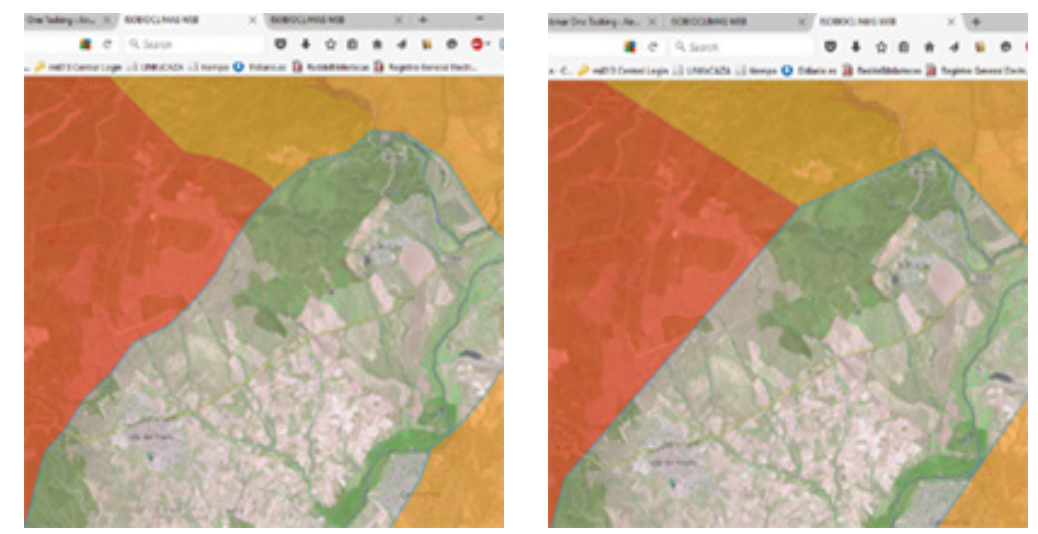

Figura 4: Detalle de la precisión en la página web completa (a la izquierda) y reducida.

Las opciones web permiten el uso de la página web si tenemos conexión a internet, pero nos puede resultar interesante la consulta del Isobioclima en campo, donde no siempre podemos disponer de conexión a internet. Para este caso se ha creado una versión consistente en un archivo KML, que se puede descargar y utilizar con o sin conexión a internet. Para utilizar este archivo debemos disponer de un programa o aplicación para que se pueda visualizar en nuestro dispositivo. Aplicaciones que manejan archivos KMZ son "Google Earth", o "Google Maps", entre otros, en el ordenador; "Google Earth", "Maps" y "Oruxmaps", en dispositivos con Android; y MotionX-GPS, en dispositivos Mac o IOS.

Las opciones para la visualización de elementos en la página aparecen arriba como tres rayitas horizontales -figura 5-.

$\mathrm{Al}$ hacer clic en las del lado izquierdo aparece una leyenda que permite activar o desactivar las diferentes capas del mapa web, la capa con la información de Isobioclimas, y las dos capas base que se pueden elegir, OSM, que es Open Street Map, un mapa topográfico con carreteras, ríos y ciudades y PNOA que es el fondo con fotografía aérea. Bajo cada una de estas capas de fondo aparece una barra horizontal con un 
cursor, moviéndolo hacia la derecha se aumenta la opacidad de la capa, si se desplaza hacia la izquierda se aumenta la transparencia.

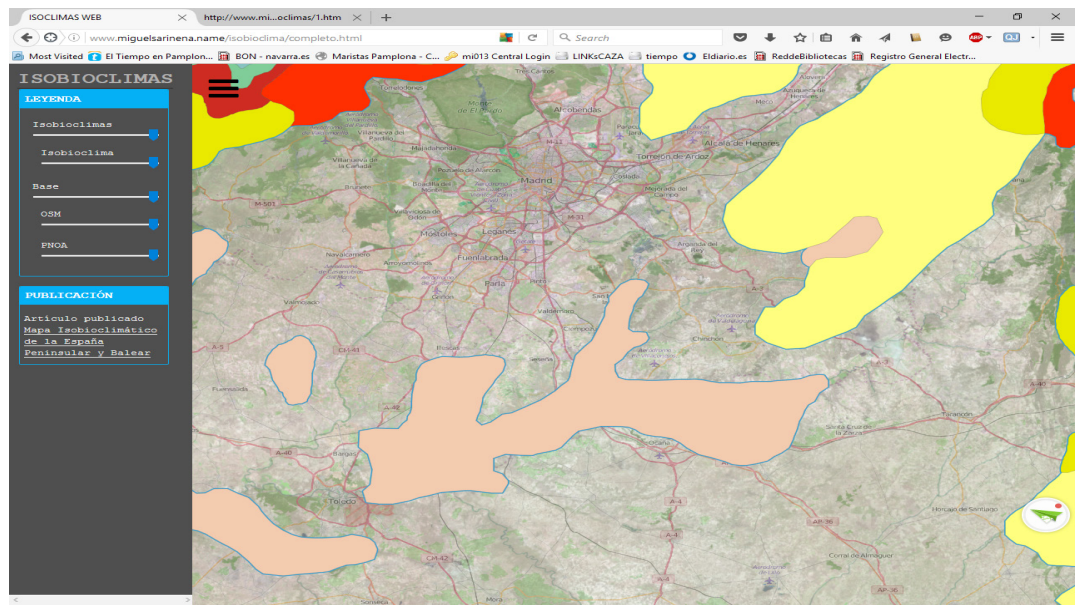

Figura 5: Funcionamiento de la página web de Isobioclimas, opciones de visualización de fondos de mapa y transparencias.

Al pìnchar sobre un isobioclima se accede a la información sobre el mismo y a un enlace web que nos ofrece más información -figuras 6 y 7 -.

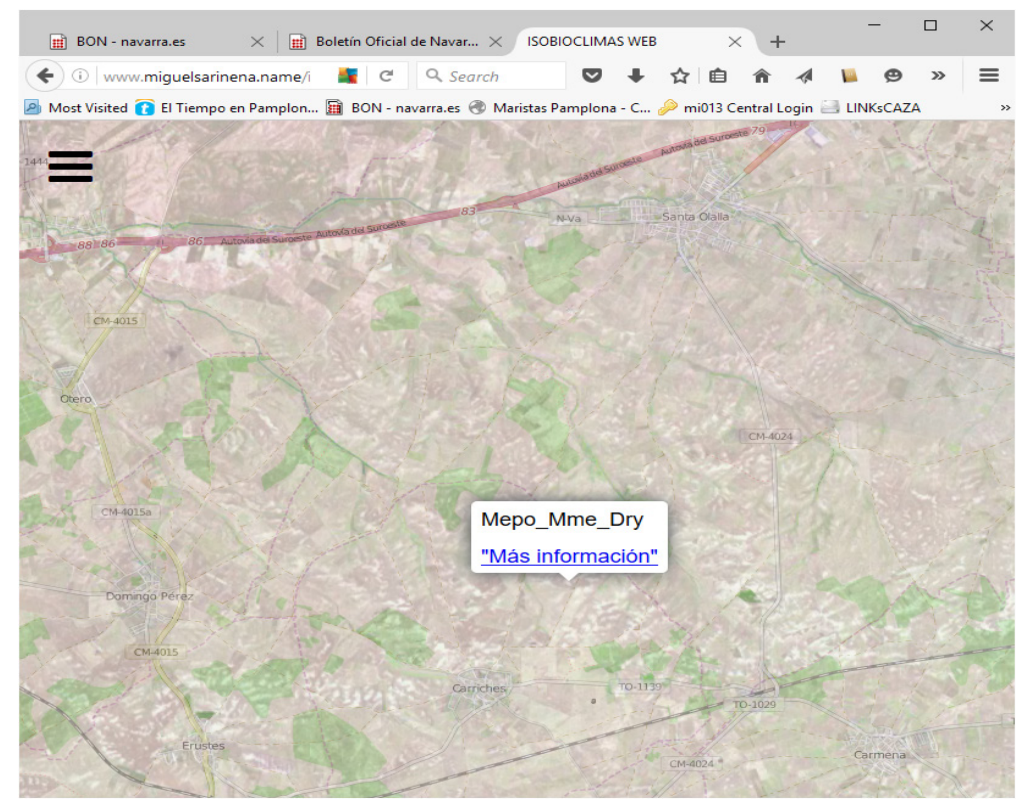

Figura 6: Funcionamiento de la página web de Isobioclimas. Información sobre los Isobioclimas. 


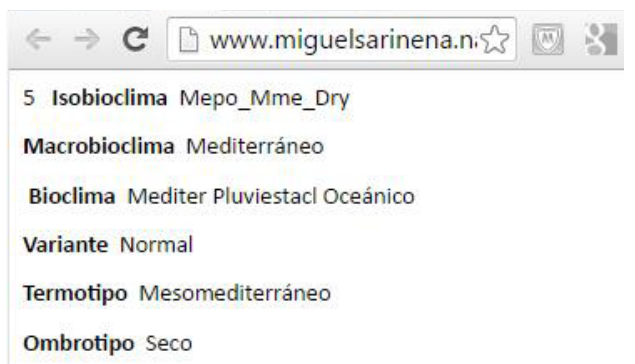

Figura.7: Funcionamiento de la página web de Isobioclimas: Acceso a más Información sobre los Isobioclimas..

La versión en KMZ -figura 8- es un archivo preparado para su visualización con dispositivos móviles o de sobremesa que tengan instalada la aplicación para manejar este tipo de archivos. Presenta la ventaja de que se puede utilizar sin conexión a internet guardando previamente la zona a visualizar en caché, tal y como se explica en la siguiente página web:

\section{https://support.google.com/earth/answer/21423?hl=es}

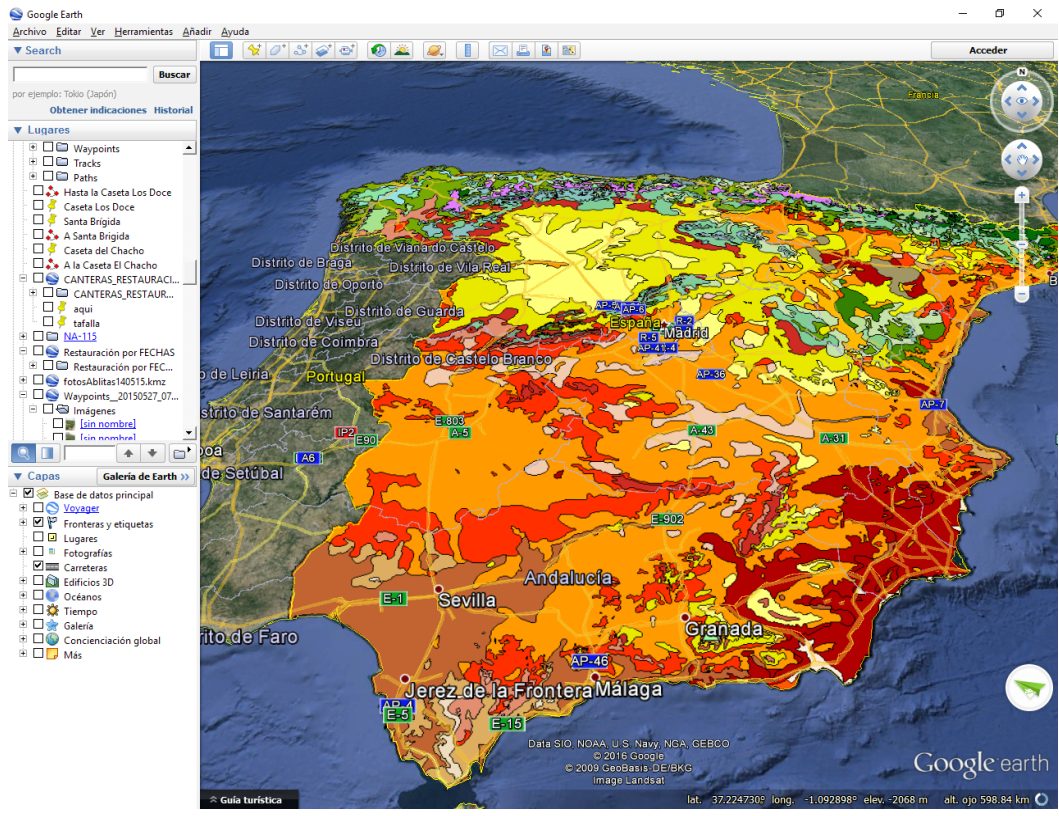

Figura 8: Visualización del KMZ con información sobre los Isobioclimas..

\section{DISCUSIÓN}

Esla primera vez que se pone a disposición del mundo científico la posibilidad de conocer, sobre el terreno, el Isobioclima reinante que se pisa, así como la extensión 
y los límites de ese Isobioclima, e incluso los Isobioclimas limítrofes, y los de toda España peninsular y Balear.

La conexión con la publicación digital originaria, López et al. 2015, permite aclarar y ampliar la información sobre cualquier Isobioclima en estudio.

Se agradecerán sugerencias por parte de los usuarios para mejorar esta página web.

A pesar de que por las herramientas web utilizadas la página web no debería dar problema para su uso en diferentes dispositivos se han detectado algunos problemas de compatibilidad con algunos dispositivos y navegadores. Algunos dispositivos I-pad, I-pad mini, o I-phone, pueden tener incompatibilidades parciales. El navegador Google Chrome, a partir de la versión 50, también puede dar problemas para aprovechar todas las características de la página web.

\section{AGRADECIMIENTOS}

Agradecemos al Profesor Rivas-Martínez, y al CIF, que nos facilitaran, en su día, la base de datos para realizar el Mapa de Isobioclimas de la España peninsular y balear, base de esta aplicación.

\section{REFERENCIAS}

Canu, S., Rosati, L., Fiori, M., et al. (2015). "Bioclimate map of Sardinia (Italy)". Journal of Maps, 11, pp. 711-718.

HTML5 https://www.w3.org/TR/html5/

López Fernández, M. L.; Marco, R.; Piñas, S.; \& López F., M.S. (2015). "Mapa Isobioclimático de la España Peninsular y Balear". Documentos Aljibe "on-line", vol. II, $n^{\text {o }} 4.7$ de octubre de 2015. Ciudad Real. Edita Sociedad Surcos. http:// www.naturalezaenhispania.com.

López Fernández, M. L.; Piñas, S.; Amézketa Ibero, A.; Aquerreta Molina, S.; López, M.S.; Almárcegui, I.; Urdiain, M.; Royo Esnal, A. (2003). "Mapa bioclimático de Navarra". Publicaciones de Biología. Universidad de Navarra, Serie Botánica. Pamplona, Volumen 15: pp 53-63.

López Fernández, M. L., Piñas, S. y López, M. S. (2008). Macrobioclimas, Bioclimas y Variantes Bioclimáticas de la España Peninsular y Balear, y su cartografía. Publ. de Biología, Univ. de Navarra Serie Botánica, Vol. 17:pp 229-236.

López, M. L., Piñas, S. y López, M.S. (2009). Isobioclimas de la provincia de Málaga y su Cartografía. En "Biogeografia Scientia Biodiversitatis" (Actas del V Congreso Español de Biogeografía. Málaga 9-12 de Septiembre (2008) Real, R \& Márquez, A.Ñ.. Editores. Málaga.

Mesquita, S. and Sousa, A.J. (2009). "Bioclimatic mapping using geostatistical approaches: application to mainland Portugal". Internatinal Journal of Climatology, 29, pp. 2156-2170.

MOBILE ENVIRONMENTAL CITIZEN SCIENCE AT MICHIGAN TECH: http:// citizenscience.cs.mtu.edu/ Citizen Science.

Mosquito alert: https://play.google.com/store/apps/details?id=ceab.move-lab.tiga-trapp. 
OpenLayers v3.15, https://www.openlayers.org

Pesaresi, S., Galdenzi, D., Biondi, E., \& Casavecchia, S. (2014).Bioclimate of Italy: application of the worldwide bioclimatic classification system, Journal of Maps, DOI: $10.1080 / 17445647.2014 .891472$

Piñas, S., López, F., M.S. y López Fernández, M.L. (2008a). Termotipos de la España Peninsular y Balear, y su cartografía. Publicaciones de Biología de la Universidad de Navarra. Serie Botánica, Volumen 17, pp 237-242.

Piñas, S., López, F., M.S. y López Fernández, M.L. (2008b). Ombrotipos de la España Peninsular y Balear, y su cartografía. Publicaciones de Biología de la Universidad de Navarra. Serie Botánica, Volumen 17, pp 243-248.

Piñas, S., López, M.L. \& López Fernández, M.S. (2009). Andalucía: Cartografía y áreas de sus Bioclimas, Continentalidad, Termotipos y Ombrotipos. En "Biogeografía Scientia Biodiversitatis". (Actas del V Congreso Español de Biogeografía. Málaga 9-12 de septiembre 2008). Ed. Real, R. y Márquez, A.L., pp. 109-119.

Rivas-Martínez, S. (2008). Globalbioclimatics, Internet: http://www. globalbioclimatics.org

Rivas-Martínez, S., Penas, A., Díaz, T.E. (2002a). "Bioclimatic Map of Portugal and Spain: 1, Bioclimates (and Variants)". Itinera Geobotanica, 15, pp. 14-15.

Rivas-Martínez, S., Penas, A., Díaz, T.E. (2002b). "Bioclimatic Map of Portugal and Spain: 2: - Thermoclimatic Belts". Itinera Geobotanica, 15, pp. 16-17.

Rivas-Martínez, S., Rivas Sáenz, S., Penas, A. (2011). "Worldwide bioclimatic classification system". Global Geobotany 634 pp + 4 Maps.

Rivas-Martínez, S., D. Sánchez-Mata \& M. Costa (1999). North American Boreal and Western Temperate vegetation. Itinera Geobot. 12: 5-316.

Scheidt, J., Koppe, C., Rill, S., Reinel, D., Wogenstein, F. \& Drescher, J. (2013). Influence of temperature changes on migraine occurrence in Germany. Int J Biometeorol. 57 (4): 649-54. doi: 10.1007/s00484-012-0582-2. Epub 2012 Aug 16.

Weather and Climate: https://play.google.com/store/apps/details?id=com.Werkzpublishing.sw.school.weather climate.

ZamiaDroid https://play.google.com/store/apps/details?id=uni.projecte. 